\title{
REUSABILITY OF IMPLANT IMPRESSION COPINGS-AN IN VITRO STUDY TO EVALUATE THE ACCURACY OF IMPLANT TRANSFER
}

\author{
Reshma Babu, Neelima Menon S* \\ Department of Prosthodontics \& Implantology PSM Dental College , Thrissur, Kerala
}

\begin{abstract}
INTRODUCTION: Implants are the mainstay of prosthetic dentistry nowadays. The number of accessory implant components essential for prosthetic rehabilitation are many. However De novo purchase of these components becomes an expensive treatment modality. So it becomes essential for the clinician to know about the reusability of these implant components without affecting the purpose served by them. Metal components are most commonly reused as they can be easily sterilized.Evaluation of reusability of impression copings on the accuracy of implant transfer. To assess the effect of multiple use of impression copings on the accuracy of direct and indirect implant transfer techniques and to assess the effect of sterilization on the accuracy of implant transfer. An acrylic resin cast consisting of five parallel holes was fabricated. Dummy implants were fixed into the holes with autopolymerizing acrylic resin. Twenty four custom impression trays (12 open trays and 12 closed trays) were fabricated. Definitive impressions were made using Vinylpolysiloxane impression material.
\end{abstract}

No statistical significance was seen between different impression techniques and each cycle of sterilization on the accuracy of impression.

Within the limitation of this study, it was concluded that the impression copings can be retrieved, cleaned, sterilized and reused upto twelve times without compromising the accuracy of the implant transfer while making impressions.

Keywords: Implants, dental impression technique, impression copings, sterilization

\section{INTRODUCTION:}

Implant therapy in dentistry is an expensive treatment modality. Fixed prosthesis supported by implants is more expensive than conventional fixed or removable prosthesis. The cost factor associated with dental implants has always been a concern among the patients, hence reusability of implant components may be desirable to reduce the overall cost of the implant therapy ${ }^{1}$.

While reusing the components both the mechanical and biological properties might get affected ${ }^{1 .}$

Metal components are most commonly reused as they can be easily sterilized ${ }^{1}$. The number of times the components can be reused safely should be determined prior to their reuse.

\footnotetext{
*Corresponding author:

Email: drneelimaprosthodontist@gmail.com

http://dx.doi.org/10.20530/IJTA 33 100-104

ISSN 2320-138X (c) 2016
}

Accuracy of implant supported prosthesis is of utmost importance to obtain passive fitting of the restoration to prevent both mechanical and biological complications associated with ill fitting restoration ${ }^{2}$.

Impression material and the technique of impression making play an vital role in accuracy of impressions for implant transfer.

Studies suggest that open tray type of impression technique is more accurate when multiple implants are placed (completely edentulous situation) and closed tray technique is preferred when less number of implants are placed and single path of retrieval of impression is possible ${ }^{8}$.

Currently square and conical form of impression copings are used in implant dentistry.

The purpose of the present short in-vitro study is to evaluate the reusability of impression copings on the accuracy of implant transfer. 


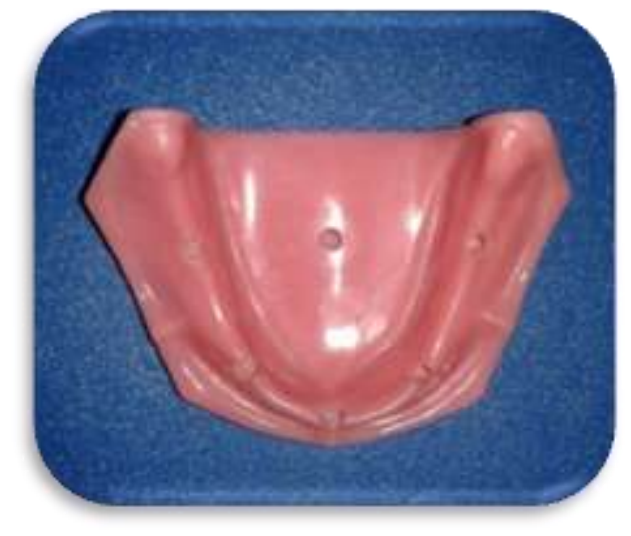

Fig 1. Acrylic resin reference cast consisting of five parallel holes $12 \mathrm{~mm}$ in length $\& 4.5 \mathrm{~mm}$ in diameter

\section{MATERIALS AND METHODS:}

\section{MODEL FABRICATION}

An acrylic resin cast was fabricated using autopolymerizing resin. Five parallel holes were drilled over the cast.A reference point was placed at the centre of the cast (Fig 1). Implant analogues were placed and fixed into the holes with autopolymerizing acrylic resin (Fig 2). Ney's surveyor was utilized to orient implant analogues vertically while placing in the drilled holes.

\section{FABRICATION OF CUSTOM TRAY}

The acrylic resin cast was covered with two layers of modeling wax to allow a constant thickness of the impression material. Four tissue stops were placed for stabilization of the tray during impression making procedure(Fig 3).Twenty four identical 2-mm-thick custom impression trays (12 open trays and 12 closed trays) were molded using auto-polymerizing resin(Fig 4,5).

Syringe loaded light body vinyl polysiloxane was placed around the implant analogues and impression copings and over the putty loaded custom tray. Two type of impression techniques were followed group

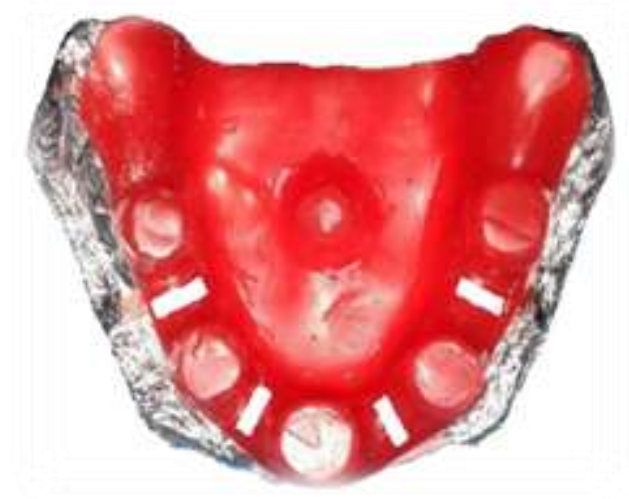

Fig 3. Fabrication of spacer

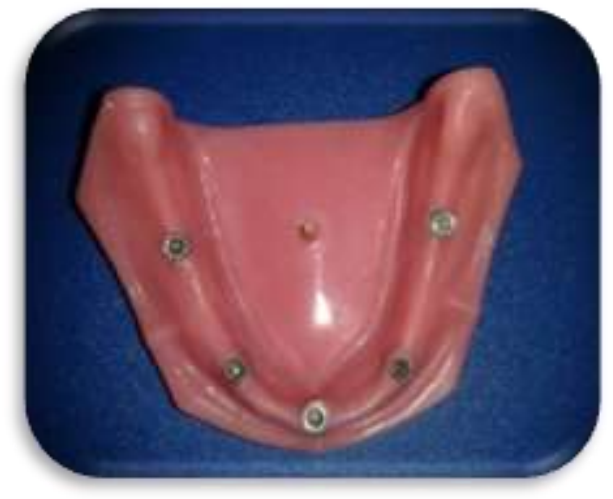

Fig 2. Five $11 \times 4.3-\mathrm{mm}$ diameter dummy implants were fixed into the holes with auto polymerizing acrylic

1(open tray technique/direct technique) group 2(closed tray/indirect technique).The impression copings were unscrewed using hex drive in case of direct technique i.e. the open tray technique after the impression material set (Fig 6). In the indirect technique i.e. closed tray technique the impression copings remained in the acrylic resin cast after the impression retrieval and then unscrewed using hex drive following which the impression copings were reoriented into the impression(Fig 7).

\section{CAST FABRICATION}

Sixty minutes after the impression procedure, the impressions were boxed. Die stone -type IV was manipulated according to the manufacturer's instructions and poured under constant vibration onto the impressions. Sixty minutes later the casts were retrieved from the impressions. Casts obtained from closed tray impression technique (Fig 8) and open tray impression technique. Co-ordinate measuring machine was used in this study. A wide, straight CMM probe recorded the distance between

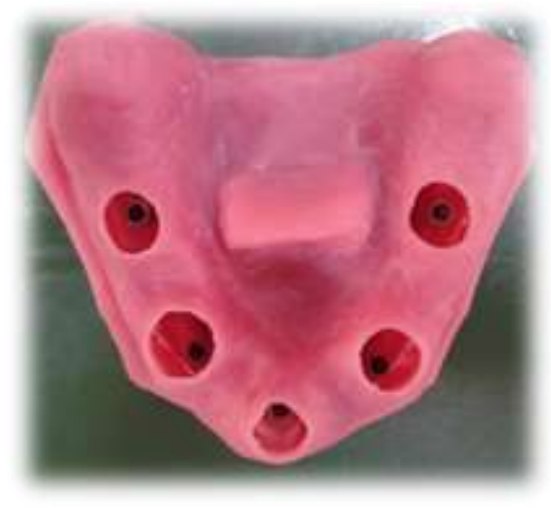

Fig 4. Fabrication of open tray 
the center of the implant orifice in each

axis ( $x$ and $y$ ) and the perpendicularity of each implant compared with the horizontal crestal plane in the acrylic resin cast.

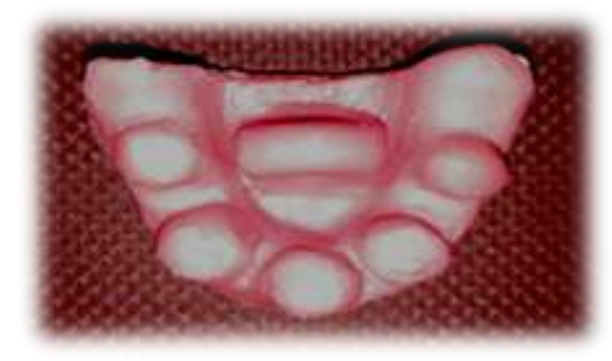

Fig:5 Fabrication of closed tray

\section{CLEANING AND STERILIZATION PROTOCOL}

As in clinical situation the impression copings were subjected to cleaning and sterilization process after every impression procedure. Cleaning procedure included scrubbing on the internal and external surface of the impression coping using soft bristled nylon brush. Followed by drying of the impression coping. Impression copings were then subjected to sterilization according to DIN standard 13060 standard protocols for sterilization of surgical and dental instruments ${ }^{1}$.The sterilization process included 10 minutes sterilization at $1340 \mathrm{c}$ and drying period of 15 minutes.

\section{REUSE PROCEDURE}

Each implant transfer procedure included making the impression, pouring the cast, removing the impression copings, and sterilizing them. The procedure was done in this way 12 times consequently, each impression coping was sterilized and used 12 times to produce definitive casts. One operator made all impressions and prepared all 24casts.

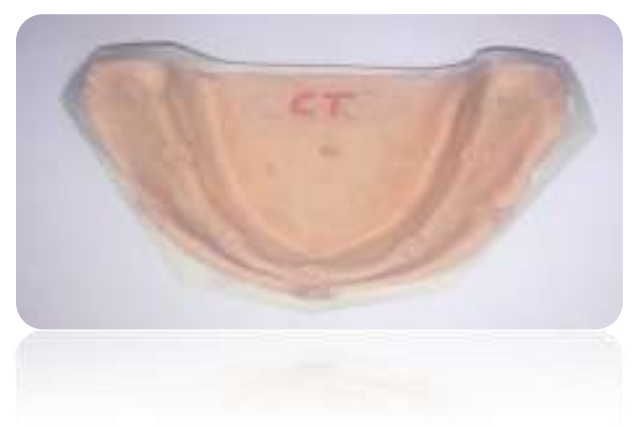

Fig 7. Casts obtained after closed tray impression technique

\section{RESULTS}

The mean value of the readings were obtained and subjected to statistical analysis (Table 1)

Student t-test was performed. Statistical significance

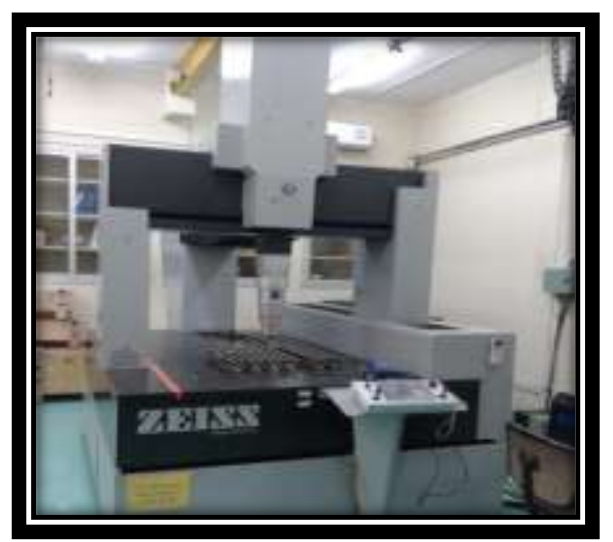

Fig 6. Co-ordinate measuring machine

was set at .05 Comparing the accuracy of casts obtained with impression copings, at the first use or during multiple reuse cycles, showed no significant differences for both impression techniques (open and closed) $(P>$.05). (GRAPH 1)

\section{DISCUSSION}

Dental care utilization is changing over the years. Recent analysis has shown that the percent of the population who see a dentist in a given year has been declining among adults and increasing among children.

Low-income adults experienced the sharpest decline in dental care utilization. More concerning is the potential effect on oral health, general health, and health care costs. Oral health is an important component of general health and routine dental care is an important component of a comprehensive oral health strategy ${ }^{3}$.

Medical devices, particularly instruments, are often replaced only when they are beyond repair.

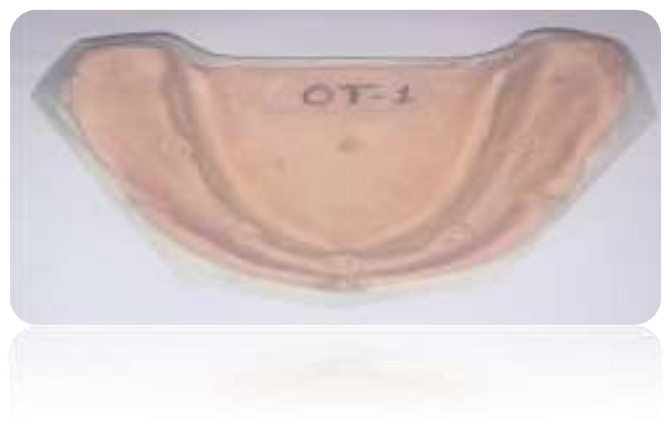

Fig 8. Casts obtained after open tray impression technique 
Consequently, older, superseded medical devices may remain in circulation long after new, redesigned instruments have been purchased. Decommissioning superseded medical devices that are difficult to clean could assist in reducing the public health risks associated with these types of medical devices ${ }^{4}$.

This study aimed at assessing the reusability of impression copings on the accuracy of implant Transfer. The results demonstrated that impression copings can be reused upto 12 times without any significant difference in their accuracy. Schwartz et al. evaluated the effects of reused cover screws on clinical outcome. It was concluded that although reusing cover screws can change their surface properties it does not affect their clinical outcomes.
Table 1. Comparison of closed tray (ct) and open tray (OT) with displacement in average of all points , A,B,C,D,E of $X$ and $Y$ planes by $t$ test.

\begin{tabular}{llllll}
\hline Variable & Group & Mean & SD & $\begin{array}{l}\mathbf{t} \text { - } \\
\text { value }\end{array}$ & $\begin{array}{l}\mathbf{p} \text { - } \\
\text { value }\end{array}$ \\
\hline X-plane & CT & 15.61 & 0.15 & - & 0.3490 \\
& OT & 15.68 & 0.20 & & \\
Y-plane & CT & 16.38 & 0.28 & 0.9323 & 0.3613 \\
& OT & 16.28 & 0.23 & & \\
\hline & & & & & \\
\hline
\end{tabular}

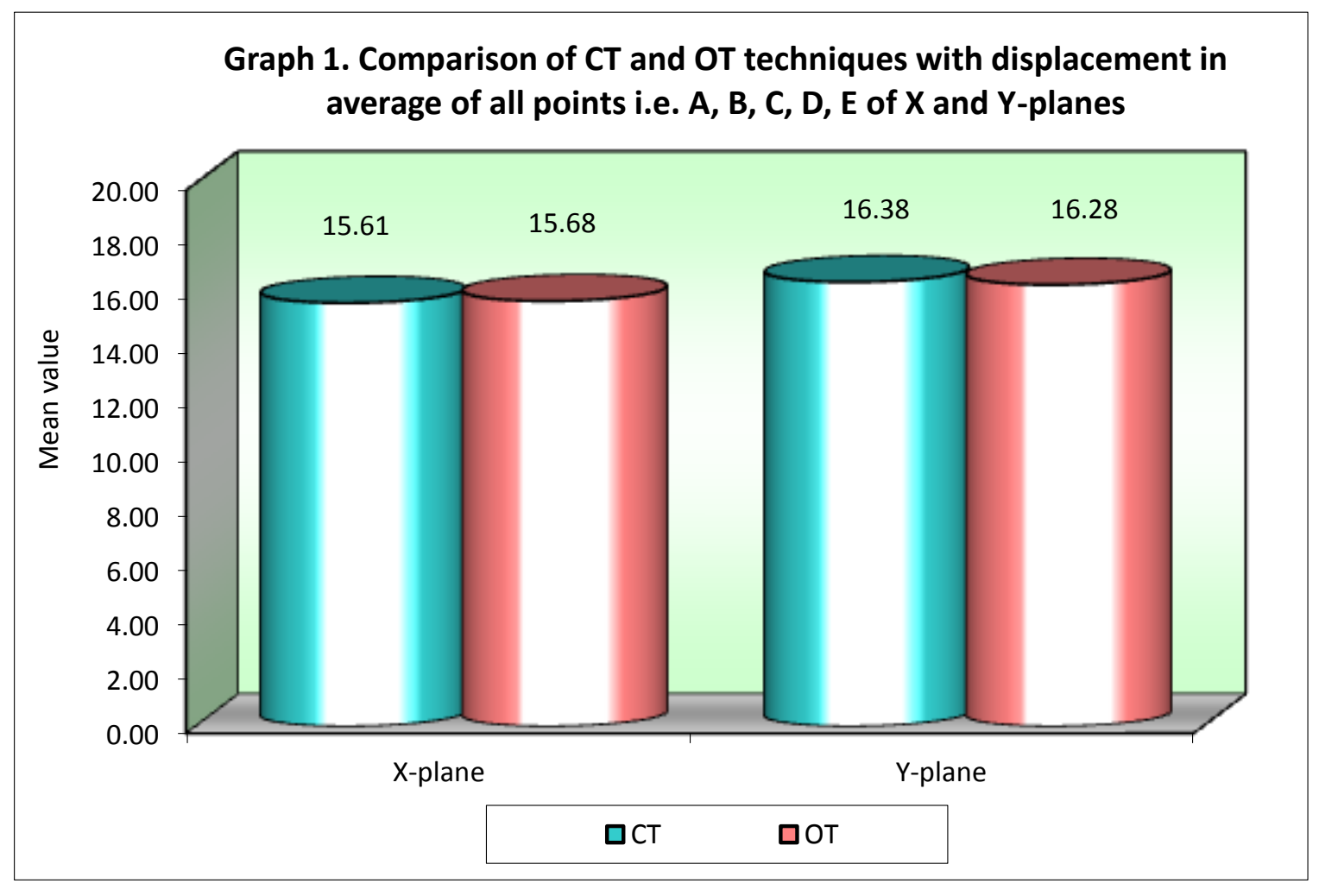

Results of the present study are in accordance to the results of the study conducted by Schwartz et al. cleaning and sterilization processes could induce thermal and chemical stresses on the material, and these stresses may lead to changes in surface morphology 5 .

Studies conducted by Martin JY et al. and Noda M et al. evaluated reuse of implant components and have shown that cleaning, sterilization, and mechanical deformations during placement and removal can alter the surface morphology of implants, which leads to changes in osteoblastic growth and differentiation. However it does not seem to be a problem with impression copings because these are temporary devices, removed after the impression procedure, and do not have any permanent contact with soft and hard tissues ${ }^{6}$.

Studies have focused on the effects of sterilization on the properties of reusable medical devices.Some of them have shown that morphologic changes 
resulting from the sterilization process could affect clinical outcomes.

Gorokhovsky et al showed that sterilization has no adverse effects on the clinical efficiency of stainless steel curettes ${ }^{7}$.

The results of present study revealed that impression copings, used during both direct and indirect technique could be reused upto 12 times without significant changes in their accuracy.

Therefore, it can be assumed that the clinical accuracy of impression copings is not sensitive to possible changes in surface properties during 12 cycles of reusing, cleaning and sterilization.

There have been several studies comparing the accuracy between direct and indirect impression technique however the rationale of this present study was not to compare these two techniques.

Several metals are used for manufacturing impression copings in different implant systems. Aluminum, stainless steel and titanium are the commonly used metals. Resistance of various alloys to the changes occurring during sterilization and reuse is not the same.

Limitations of this study includes less sample size, use of one implant system and use of only square type of impression copings.

Further investigations are required to determine the number of episodes the impression copings can be reused without compromising the accuracy of impression, and to distinguish if any change in the surface property is related to impression process, sterilization or both.

\section{CONCLUSION}

Within the limitation of this study, it was concluded that the impression copings can be retrieved, cleaned, sterilized and reused upto twelve times without compromising the accuracy of the implant transfer while making impressions.

\section{REFERENCES}

1. Alikhasi M, Bassir SH, Naini RB. Effect of Multiple Use of Impression Copings on the Accuracy of Implant Transfer. JOMI; 2013 Mar;28(2):408-14. Available from: http://dx.doi.org/10.11607/jomi.2717

2. Sorrentino R, Gherlone EF, Calesini G, Zarone F. Effect of Implant Angulation, Connection Length, and Impression Material on the Dimensional Accuracy of Implant Impressions: An In Vitro Comparative Study. Clinical Implant Dentistry and Related Research;
2009 May 12;12:e63-e76. Available from: http://dx.doi.org/10.1111/j.1708-8208.2009.00167.x

3. Vujicic M, Nasseh K. A Decade in Dental Care Utilization among Adults and Children (2001-2010). Health Services Research; 2013 Dec 3;49(2):460-80. Available from: http://dx.doi.org/10.1111/1475$\underline{6773.12130}$

4. The Therapeutic Goods Act of Australia. International Labeling Requirements for Medical Devices, Medical Equipment and Diagnostic Products; 2003 Jun 27; Available from: http://dx.doi.org/10.1201/9780203488393.pt3

5. Schwartz Z, Lohmann CH, Blau G, Blanchard CR, Soskolne AW, Liu $Y$, et al. Re-use of implant coverscrews changes their surface properties but not clinical outcome. Clin Oral Implants Res; 2000 Jun;11(3):183-94. Available from: http://dx.doi.org/10.1034/i.16000501.2000.011003183.x

6. Martin JY, Dean DD, Cochran DL, Simpson J, Boyan BD, Schwartz Z. Proliferation, differentiation, and protein synthesis of human osteoblast-like cells (MG63) cultured on previously used titanium surfaces. Clin Oral Implants Res; 1996 Mar;7(1):27$37 . \quad$ Available from: http://dx.doi.org/10.1034/i.16000501.1996.070104.x

7 Sahrmann P, Ronay V, Hofer D, Attin T, Jung RE, Schmidlin PR. In vitro cleaning potential of three different implant debridement methods. Clin Oral Impl Res; 2013 Dec 26;26(3):314-9. Available from: http://dx.doi.org/10.1111/clr.12322

8. Papaspyridakos P, Chen C-J, Gallucci G, Doukoudakis A, Weber H-P, Chronopoulos V. Accuracy of Implant Impressions for Partially and Completely Edentulous Patients: A Systematic Review. Int J Oral Maxillofac Implant; 2014 Jul;29(4):836-45. Available from: http://dx.doi.org/10.11607/jomi.3625 